\title{
Develop a Model for the Elders' Leisure Time
}

\author{
Mohammad Hosein Ghorbani ${ }^{1} \&$ Seyede Tahereh Mousavirad ${ }^{1}$ \\ ${ }^{1}$ University of Tehran, Tehran, Iran \\ Correspondence: Mohammad Hosein Ghorbani, University of Tehran, Tehran, Iran. E-mail: \\ H_Ghorbani91@yahoo.com
}

Received: September 19, 2016

Accepted: October 2, 2016

Online Published: February 22, 2017

doi:10.5539/res.v9n1p279

URL: http://doi.org/10.5539/res.v9n1p279

\begin{abstract}
The present research intends to examine the model for elders' leisure time. This research is a descriptive-analytical study, reported as applied study. The statistical population consists of the faculty members of universities, managers and specialists at Championship Deputy, General Sports Federation and rural sports federation and the sociologists. To analyze data, both descriptive and inferential statistics were used. In the present research, software SPSS22 was used for structural equation and software AMOS was used for design of model. Results from research led to detect eight factors including managerial-political, economic, sociocultural, health, educational, facility, sports, individual-environmental factors affecting elders' leisure time. Findings from confirmatory factor analysis indicated that the elders' leisure time can be used. To sum up, the results from research led to design of model for elders' leisure time so that managerial-political, economic, sociocultural, health, educational, facility, sports, individual-environmental factors with factor loading $0.9,0.87,0.85,0.84$, $0.82,0.76,0.71$ and 0.63 have the highest contribution, respectively. Further, findings from model fitting indices indicate favorable fitting and confirmed model for elders' leisure.
\end{abstract}

Keywords: leisure time, elders, model

\section{Introduction}

Aging population refers to one of the phenomena of our time with the least deal with it, accurate progress, the best status in future as forecasted and the most outcomes. This goes true in Iran, like many countries, and Iran's movement to an aging population can be seen clearly. Hence, we will face a high percent of elderly population in near future, thus planning to resolve predicted and unpredicted problems and meeting needs of this population group should be considered at the study works and major plannings of the country (Imani, 2011). Of the issues that demographers and social pathologists have attempted in the past years to confirm warnings about it refers to the phenomenon of middle-aged population that can be considered a risk for a country, because with regard to the statistics in 2016, Iran population will enter to the middle-aged stage, while as said by the experts, Iran has not suitable structures in terms of arrival to the aging period, for which planning should be considered based on population forecasts about the elders' needs including nursing homes, Food industry in accordance with the elders' gastrointestinal tract, elders' leisure time and so on (Vakili, 2011). On the other hand, leisure time is the most important and pleasant time for human beings and a part of hours in human's daily life that the person engages in rest, leisure, development of awareness or social participation and engages in them voluntarily after liberation from the job, family and social requirements. Leisure can be considered as the most important opportunities and hours in human life (Sanaei, 2013). One of the most important causes of deviance, delinquency and social distortions is the personal and social disorganization in the field of leisure. From Islamic perspective, leisure time is neither the time of liberation from legal and ethical constraints nor time of irresponsibility (Sharifi, 2012). Studies indicate that individuals in Iran society have free time for 3-4 hours in average in 24 hours a day. In other words, one sixth or one third of the individuals' lifetime should be planned with the suitable and useful programs separated from the daily life programs to spend leisure time of different social classes. Leisure in general is a new concept, born out of the industrial age to which various responses have been given at various communities well suited to cultural conditions, extent of development and social welfare, so that specific facilities are prepared and various mechanisms well suited to the community are designed. Nowadays, countries invest in spending leisure time as investing in employment. With regard to the necessity and importance of organizing leisure activities for elders, most of developed countries have made macro and systematic planning on how this group of people over 55 years old spend their leisure time (Jahromi, 2012). However leisure and recreational sports 
management is important in an appropriate way for all segments of society, and decisions and planning should be made in accordance with conditions, features, facilities, tendencies and tastes of individuals, two groups of people - Disabled and Elderly - need a special attention and different outlook. What makes the importance of leisure in the elderly population: 1) elderly have more free time than others, that is, free time should not be confused with leisure time; 2) unfortunately little attention is paid to them in society. This can be witnessed among the family members who assume that the elders have no longer any needs and just have to sit in the corner waiting for death; 3) leisure activities and especially recreational sports are far more important to them than other people; 4) With increasing age, attention and sensitivity to health increases; 5) it should pay attention to the fact that most of elders have gained valuable experiences in their lifetime. They enjoy numerous abilities, worked in important jobs. Now if the elders fail to use their experiences and provide them for others, they will suffer from mental and emotional problems and the community will be damaged. For this, it requires making a specific planning for elders' leisure to convert their free time to fruitful leisure time for themselves and other people in the society (Farahani, 2012). In the elderly, many people suffer from tough things in life such as retirement, financial problems, loss of health, and death of a spouse or friend that can damage their social network.

This damage is particularly disturbing when more senior citizens need social support to help identifying and fixing problems and need preparing immediate needs and facilities to get help from external resources. To encourage seniors to participate in a planned and organized way, a well-planned policy is required. It cannot forget that the elderly have valuable contribution to the country, grown regarding their enriched knowledge and experience during active participation in lucrative jobs. Thus young generation must know respecting to the elderly and supporting them are their duties (Jogender, 2013). Population aging is the effect of the demographic transition, while the western countries have experienced this process and have planned for their elderly. That is why Asian countries have faced constant growth in elderly population in the late one and half past century ago which have led to decrease of mortality and better healthcare and improvement in total quality of life. Thus there is an immediate need to examine various aspects of this growing population, dependent on suitable design of the associated policies and programs to resolve needs of this vulnerable group. The elderly process has been witnessed with high speed in developing countries. The studies in this context to provide the proposed necessary factors have been regarded as the suitable policies for the growing population especially due to social welfare (Kiran, 2015). Indeed, any effort by today's generation to improve quality of leisure time for the elderly at dimensions of leisure programs and place features will be a planning for future of today's generation who reach to the elderly (Ghanbarian et al., 2011).

Discussion on filling the optimal time is important as this time is like a double-edged sword, that is, the edges can be reformative and growing and other edges can be undermining the power and even the futility and corrupt. For this, it can say that how spending leisure time even from positive and constructive aspects and from negative and corruptive aspects is to the extent that affects other life aspects of any person in a large extent (Karimian, 2009). Regular and light exercises that increase heart rate and breathing have been regarded as the critical factor to maintain the health of the elderly. Authentic studies have shown that regular and light exercises postpone the process of aging (Mac Glayn, 2006). The government can avoid the deviant behaviors or crimes with exercise and leisure time for the elderly. Government not just can help to reduce deviant behavior and crime in the elderly but also can help to create a long-term program to avoid committing crime for the elderly (Chan-Woo et al., 2014). Joint activities will allow the elderly to learn from each other many problems. Further, exchange of information in addition to improvement in relationships causes learning new technologies and problems. It is hoped that the role of government especially community leaders in macro outlook to the elderly's leisure time is highlighted and planned for it in a way to cause improvement in physical and mental health (Bahram, 2013). Today spending leisure time is a problem which has preoccupied mind of most of politicians and planners around the world. What role the leisure time has in the people's life especially the elderly's life has been elaborated many times, while the communities which understand this role better act more successful in their plans. Leisure time is of great importance to all the age groups and the elders are among the classes in the community who need far more attention. Nowadays, almost all over the world believe that respecting the elders and maintaining their high social status should be considered a standard in programs of government and society to preserve and strengthen national and cultural interests, thus the officials of sociocultural programs and sustainable development of countries are assigned with grounding the support programs for the elderly on recognizing the elders' problems and resolving their problems properly so as to achieve transition of their valuable experiences to young generation. With regard to the elderly population growth and increase in life expectancy due to the advancement of issues in health and medicine, the necessity to attention to the problems and needs of this generation grows and planning necessitates for them, because on one hand the seniors are the treasures of any society which these treasures are replete with experience, skill and efficiency, so that any society is assigned with maintaining their treasures and utilizing them 
for investing in this way; on the other hand, by increasing age, the risk of complications and physical and mental disorders increases in the elderly which this will impose huge costs on families and society. Thus it seems wise that any society before facing a critical situation from aging at society predicts the required conditions and facilities and plans for this. How to spend leisure time seems a serious issue to which a particular attention should be paid, because this time under proper planning will not just remain useful but also it will include many material and spiritual interests (Chaman, 2010). Researchers have conducted few studies on elders' leisure time. Madah et al. (2009) examined study on status of social activities and how elders spend their leisure time in Iran and Sweden. The factors including development of urbanization, lack of facilities, and lack of familiarity with attention to healthy lifestyle have caused lesser Iranian elders' satisfaction with social relationships and how to spend leisure time than Sweden elders. On the other hand, attention to spiritual dimension of health in Iranian elders has been higher, found with a significant difference with Sweden elders, while this relationship is inverse and significant on group activities. Further insufficient attention to physical activity and exercise especially in Iranian elder women is a point which must be taken into account by the planners and politicians at health area. Singh and Keiran (2015) examined effect of educational status on the elders' welfare, which the results indicated that the elders' educational status affects their welfare, so that the elders with better educational status enjoyed higher social welfare.

Su et al. (2014) examined effect of participation in recreational activities on mental and physical health of elders which the results indicated that: 1) participation in recreational activities puts a positive effect on physical and mental health of elders, 2) elders' physical and mental health affects their quality of life, 3) physical and mental health affects the relationship between participation in recreational activities and elders' quality of life. Further the results indicated that major human activities should centralize on participation in recreational activities. Far more emphasis has been put on mental health for the elders; social interactions have been regarded as the major aspects in quality of life of the elders. The results from findings of research by Raein et al. (2015) indicated that there is a positive significant relationship between leisure time activities and recreational activities which are grounded on physical activity; leisure time activities increase happiness and feeling of pleasure in the person. Further the results indicated that there is a positive significant relationship between leisure time activities and feeling of self-efficacy. The results from the study by Tribees et al. (2012) indicated that the physical activity during the leisure time and total physical activity cause lack of weakness in the elderly. Due to the necessary size to prevent weakness, it is suggested that men should have moderate to severe physical activity for 140 minutes per week and women should have moderate to severe physical activity for 145 minutes per week. Similarly women should have physical leisure activities for 85 minutes per week and men should have it for 112.5 minutes per week, which this is sufficient for lack of weakness in the elderly. Rahimi et al. (2010) examined the elders' experiences in Tehran in the context of leisure time and recreations, which the results indicated that the elders do not enjoy the satisfactory leisure pattern regarding the participants' experiences on the concept under study. The results from study by Lee Chungsup et al. (2015) can be mentioned in this way that addressing the leisure activities is far more important than addressing other special activities in the successful elderly. The policy and planning related to leisure cannot be considered regardless of other social conditions and as a policy separate from culture, economy, facilities and educational status of society, thus the researchers intends to give a response to this question "how is the model of leisure for the elders?".

\section{Research Method}

The research method is a descriptive-analytical method; this research is an applied study in which the elders' leisure has been modeled. The statistical population consists of the faculty members of universities, managers and specialists at Championship Deputy, General Sports Federation and rural sports federation and the sociologists. Data collection instruments include 1 research-made questionnaire consisting of 77 questions prepared to model and determine factors affecting elders' leisure time. In this study, content and face validity has been confirmed by 15 experts. Factor analysis was used for the construct validity and reliability of the questionnaire was calculated via Cronbach's alpha equal to 0.83 . In the present research, both descriptive and inferential statistic methods have been used. In the present research, firstly demographic characteristics in the research samples have been described and Kolmogorov-Smirnov test was used to examine normality of data. Factor analysis will be used for the test. In the present research, software SPSS22 was used; further software AMOS was used for structural equations and design of model.

\section{Results}

With regard to the research goals and questions, firstly the descriptive information on the research samples have been described based on variables of gender and education and then findings of the present research in form of response to the research questions have been proposed. 


\subsection{Descriptive Findings}

Findings indicate that 35, 80 and 185 persons in the sample groups have bachelor degree, master degree and $\mathrm{PhD}$, respectively. Further findings indicate that 202 and 98 persons are men and women, respectively.

\subsection{Normal Distribution of Data}

Table 1. Kolmogorov-Smirnov test to examine normality of data

\begin{tabular}{cccccc}
\hline Questionnaire & No & Mean & Standard deviation & k-s Statistics & Sig \\
\hline Factors affecting elders' leisure time & 300 & $3 / 52$ & $0 / 16$ & $3 / 38$ & $0 / 431$ \\
\hline
\end{tabular}

With regard to the results from Table 1, it can conclude that as value of sig for the questionnaire are greater than test level $(\alpha=0.05)$, all the variables of the questionnaire enjoy normal distribution.

\section{Recognizing Factors Affecting Elders' Leisure Time}

Results indicate that all the required assumptions relating to use of factor analysis method has been considered. Kaiser-Meyer-Oklin test is an index for the sample adequacy. With regard to the mentioned test, it can recognize the extent to which the variables belong to each other and their appropriateness for factor analysis and also it can specify appropriateness of each variable. In Bartlett's test of sphericity, the correlation between questions was examined. With regard to the $\mathrm{k}$-square value and sig level, it can conclude that there is correlation between questions. Thus use of other stages of factor analysis is allowed. With regard to the results from the initial estimation for the common point in variables, their valued equaled to 1 . The obtained values indicate the factor correlation. Since the common variance of all the items has been reported greater than 0.5 , all the items are used for factor analysis. The predictability power of this model based on sum of percent of cumulative variance of factors equaled to $76.42 \%$. Therefore, with regard to the results, eight factors including managerial-political, economic, sociocultural, health, educational, facility, sports, individual-environmental factors were found as the factors affecting elders' leisure time.

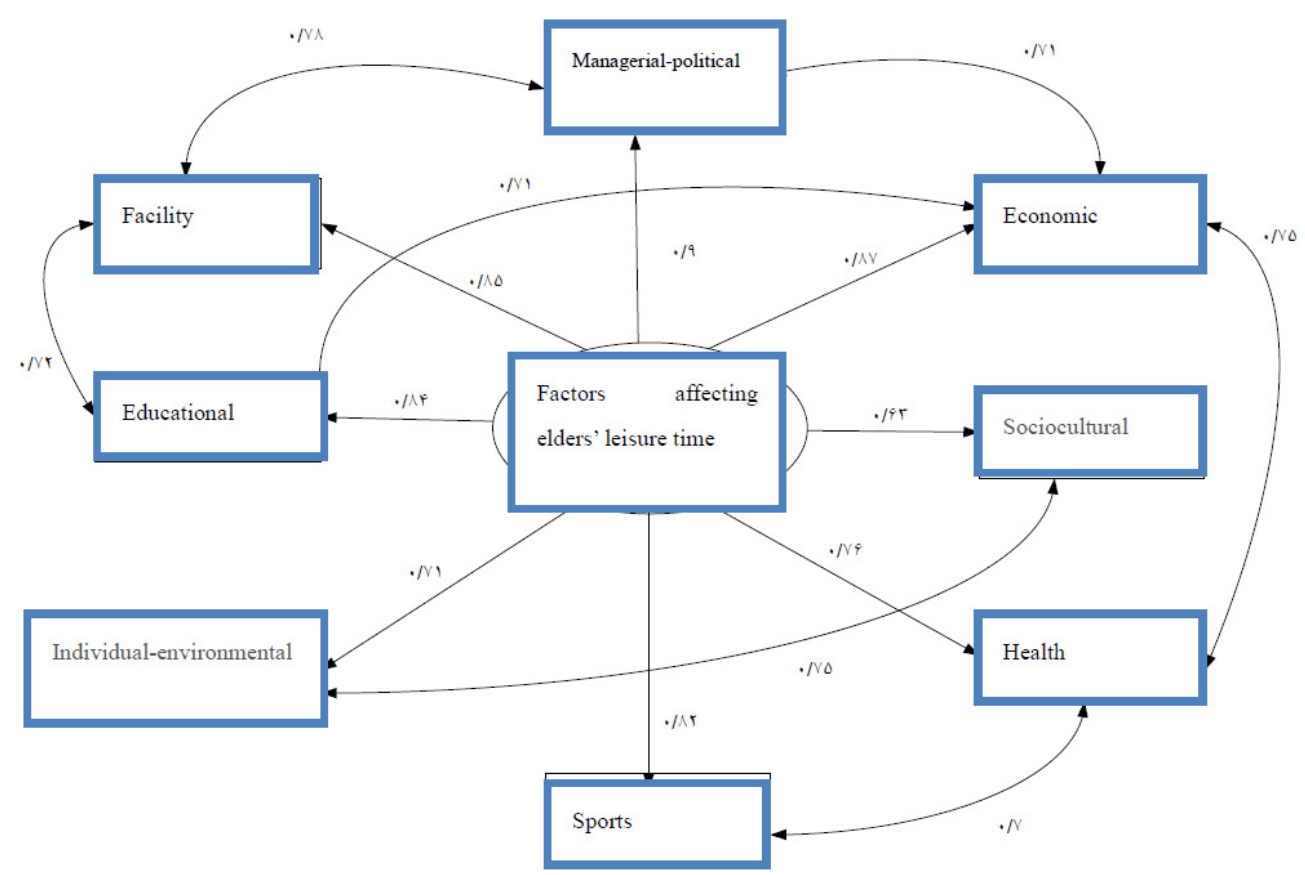

Figure 1. The model for factors affecting elders' leisure time 
As shown in Figure 1, in the model for the factors affecting elders' leisure time, managerial-political, economic, sociocultural, health, educational, facility, sports, individual-environmental factors with factor loading $0.9,0.87$, $0.85,0.84,0.82,0.76,0.71$ and 0.63 have the highest contribution, respectively. Further, there is a mutual relationship between managerial-political and facility factors, managerial-political and economic factors, facility and educational factors, economic and educational factors, health and sports factor, sociocultural and environmental-individual factors. To test whether the factors affecting elders' leisure time enjoy suitable fitting, model fitting indices were used. In structural equation modeling with an emphasis on software AMOS, three indices including Absolute Index, Comparative Index, Parsimonious Index should be used to determine model fitting. With regard to the results from table, value of $x^{2}$ equaled to 1.59 ; since this value is between $1-3$, this indicates confirmed model. Further, p-value equals to 0.69 which is greater than 0.05 , thus the model is confirmed. Goodness Fit Index equals to 0.9 indicating acceptability of this value for the proper model fitting. Root Mean Squared Error of Approximation which is another index of Goodness Fit Index equals to 0.001 indicating confirmed model. Other indices of Goodness Fit Index for structural equations include Tucker-Lewis Index (0.92), Bentler-Bonett Index (0.94), Comparative Fit Index (0.9) and Parsimonious Normed Fit Index (0.09), indicating proper fitting and confirming the model for factors affecting elders' leisure time. Results from Friedman test to determine difference of rank for the factors affecting elders' leisure time indicated that value of $\mathrm{k}$-square $\left(\mathrm{x}^{2}\right)$ equals to 47.37 . With regard to value of $\mathrm{x}^{2}$ and $\mathrm{P}<0.05$, it can conclude that there is a significant difference between mean of rank for the factors affecting elders' leisure time, which the ranks include managerial-political, economic, sociocultural, health, educational, facility, sports, individual-environmental factors.

\section{Discussion and Conclusion}

Nowadays, needing to plan for leisure time for people is of great importance, because if the leisure time is used properly, this will cause the development for the person, otherwise many problems will raise for the people. The main purpose of the present research was to examine the model for the elders' leisure time. The results from the present research indicate that eight factors exist as the factors affecting elders' leisure time, including managerial-political, economic, sociocultural, health, educational, facility, sports, individual-environmental factors; these results are consistent with the Seniors Strategy Town of Whitby's and Older Adult Strategy Town of Milton. The results of research to determine the model for the factors affecting elders' leisure time indicated that in the model for elders' leisure time, managerial-political, economic, sociocultural, health, educational, facility, sports, individual-environmental factors with factor loading $0.9,0.87,0.85,0.84,0.82,0.76,0.71$ and 0.63 have the highest contribution, respectively. This finding of research indicates that an effort to improve and determine political and managerial factors will have more effects than other factors to improve and develop elders' leisure time, and it can raise favorable conditions for leisure time of this class by implementing suitable policies and monitoring elders' leisure time, deduced that strategic planning for elders' leisure time has the highest contribution in this context. Further, in the mentioned model, there is a mutual relationship between managerial-political and facility factors, managerial-political and economic factors, facility and educational factors, economic and educational factors, health and sports factor, sociocultural and environmental-individual factors. Further there is an indirect relationship between political-managerial and educational factors, political-managerial and health factors, facility and economic factors, sport and economic factors. Since such statement has been examined for the first time, the evidences and works similar to it have not been found. A particular attention should be paid to the factors which have a mutual significant relationship with each other in the planning, because suitable or unsuitable activity in a factor affects another factor, thus a special attention should be paid to the factors. Further the factors which have an indirect relationship with each other have influenced on each other so that their influenced on each other more likely emerges in long term. Various steps of this model have been determined based on the preferences from the research findings, found as the reflection in the experts' proposed programs. Using the proposed model, it can have the most utilization with a systematic outlook toward the elders' leisure time and resolve the weaknesses, cope with threats and strengthen the opportunities and strengths. The results from this research can be used by the planners and practitioners in the context of leisure time especially the elders' sector. Further the results can be used for different group of people, organizations and entities including Leisure and recreation organization, top managers, planners and policy makers and administrators in the Seniors \& Retirement leisure sector, Civil Servants Pension Organization, National Seniors Council, Ministry of Labour and Social Welfare, Welfare Organization, Social Security Administration, the nursing homes in the country, NGO operating in the elderly such as Seniors' Health Support Foundation, Ministry of Labour and Social Affairs, Imam Khomeini Relief Committee and Municipalities. The results from research can be used in formulating Strategic plans, benchmarking, compensation decisions and leisure services for the elderly. Results from research can be used in preparing short-term, medium-term and 
long-term plans as well as creating or modifying the elderly and retirement policies. This model assists for describing the relationships between the elements inside the factors affecting the elders' leisure time. Further, reducing disability and increasing quality of life, successful elderly, active elderly, skill and ability, feeling of self-esteem, good spirit, joy of life, helping to increase the presence of the elderly and the moving towards community development, reducing disease, improving physical, mental, social and spiritual health of elders and reducing the elderly risks, creation the conditions to make social relationship for elders and expanding the culture of respect to the elders, increasing employment and improving the living spaces for the elders can be made by implementing model programs. Further, this can be used for other modeling. In general conclusion, it can say that development of elders' leisure has been influenced of various factors affecting the elderly; in this research, development of elders' leisure has been under influence of political-managerial, economic, sociocultural, health, educational, facility, sport and environmental-individual factors. As a result it seems that the required infrastructures together with educational planning should be considered for the factors affecting leisure time so as to reach the leisure time. Before conducting this research, knowledge on use of this model and its applications as well as its components was not existed, but conducting this research revealed that the model for elders' leisure can be a new model and pattern to develop elders' leisure. Results from this research can have practical applications for the leisure time's practitioners.

\section{Executive and Research Suggestions and Strategies}

1) Acknowledge the active and superior elders, i.e., the elders who gain honors in the scientific and sport areas and are suitable model for other elders.

2) Model the elderly sports in developed countries and create an infrastructure and provide the environment for these sports such as Pickleball, Short Tennis and Walking Football.

3) Elderly insurance is a controversy in our country that modeling the insurance plans in developed countries or creating a smart plan well suited to the elders' status can be used to remove this challenge.

4) Employ the specialists at leisure time by the organizations and custodians can reduce many leisure problems of elders and other classes.

5) Attention to elders' various leisure needs and ethnical, regional and language differences and make suitable strategies for each is of great importance in this context.

\section{References}

Amir, B. M. (2013). Recommendations and warnings by Dr Ali Baghbanian to fill the leisure time of the elderly. Health Newspaper, 438.

Chamanara, M. (2010). How elders spend leisure time with an emphasis on physical activity and their satisfaction with municipal sports facilities?

Chan-Woo, A., Eun-Surk, Y., Dong-Kyun, P., Hi-Wan, L., Kwang-Uk, L., Byeong-Seok, M., ... Ik-Young, C. (2014). A study on the development of the protocol of leisure activity program for the elderly's deviant prevention. Journal of Exercise Rehabilitation, 10(2), 106-110. https://doi.org/10.12965/jer.140105

Ghanbarian, S., Varesi, H. R., \& Mohammadi, H. (2011). Evaluation of site for elders' leisure time with an emphasis on individual factors-Case study: Areas 3 and 5 Isfahan. Journal of Geography and Environmental Planning, 22(42).

Jalali, F. M. (2012). Leisure and Recreational Sports Management (7th ed.). Tehran University Press.

Jahromy, N. (2012). Principles of Sociology in Sport (2nd ed.). Publications of Bamdad Ketab.

Karimiyan, H. (2009). A look at the Leisure. Fields and Practices, Knowledge Eighteenth Year, 141, 111-126.

Lee, C., \& Laura, L. (2015). Exploring the Relationship between Different Types of Serious Leisure and Successful Aging, 16.

Mac Glayn, G. (2006). Dynamic physical fitness (1st ed.). Chakameh publishing.

Madah, B. (2009). The state of social activities and how to spend leisure time in the Iranian and Sweden elderly. Iranian Journal of Ageing, 8(12).

Raein, P., Zarei, A., \& Hami, M. (2015). The Impact of Sports' Leisure Activities on Happiness and Self-efficacy Among University of Sari Students. International Research Journal of Management Sciences, $3(1), 24-28$. 
Rahimi, A., Anousheh, M., Ahmadi, F., \& Foroughan, M. (2010, Spring). Elders' experiences in Leisure and entertainment. Iranian Journal of Ageing, 9.

Recreation and Leisure Services. (2014-2018). Seniors Strategy.

Sanaei, N. (2013, spring and summer). Conceptual geometry for leisure time with sociological, psychological, moral approach, fourth year, 1, 93-111.

Sharifi, A. H. (2012). Marigold. In Islamic ethics and lifestyle. Qom, Education.

Singh, B., \& Kiran, U. V. (2015, January). Impact of Educational Status on Social Well Being of Elderly. International Journal of Research, 2(1).

Singh, J. (2013, May). Aged in the Family: A Sociological Study in Haryana. Department of Sociology Maharshi Dayanand University Rohtak, Regn.

Su, C. L., Lee, C. J., \& Shinger, H. S. (2014). Effects of Involvement in Recreational Sports on Physical and Mental Health, Quality of Life of the Elderly. Anthropologist, 17(1), 45-52.

Tribees, S., Jair, S. V., \& Ricardo, J. D. O. (2012). Physical activity as a predictor of absence of frailty in the elderly.

Vakili, M. A. (2011). Age transition of Iran to Chel Celli period. Ebtekar Press.

\section{Copyrights}

Copyright for this article is retained by the author(s), with first publication rights granted to the journal.

This is an open-access article distributed under the terms and conditions of the Creative Commons Attribution license (http://creativecommons.org/licenses/by/4.0/). 